\title{
EMPRENDIMIENTO DOCENTE EN LA TRANSICIÓN DE EDUCACIÓN PRIMARIA A EDUCACIÓN SECUNDARIA: UNA PROPUESTA DESDE LA EDUCACIÓN DEPORTIVA Y EL APRENDIZAJE-SERVICIO
}

\author{
Luis Miguel García López \\ David Gutiérrez \\ Juan Gregorio Fernández Bustos \\ Universidad de Castilla La Mancha
}

RESUMEN: La transición de Educación Primaria (EP) a Educación Secundaria (ES) es un periodo perturbador y de gran influencia en la trayectoria escolar de más del 50\% de los estudiantes, con una disminución sustancial en la autoestima, la motivación académica y el logro académico, así como el riesgo de sufrir conductas de acoso y abandono escolar. Los sistemas desarrollados habitualmente para facilitar este proceso se han centrado en los procedimientos administrativos y de organización, cuando las investigaciones muestran que el proceso debería centrarse en los aspectos sociales y personales. En este trabajo exponemos cómo la utilización integrada de la Educación Deportiva y el Aprendizaje-Servicio puede configurarse como una potente herramienta para combatir los problemas derivados de este proceso de transición educativa. De esta manera, analizamos cómo la implementación conjunta de estos dos planteamientos permite contar con facilitadores de gran potencial en este proceso: tener conocidos que pasan con ellos de EP a ES, desarrollar el sentimiento de pertenencia, conocer a alumnos mayores y comprobar que son amistosos, realizar actividades de acogida que les ayuden a adaptarse y percibir que el centro les ayuda, y actividades en grupos reducidos donde puedan relacionarse estrechamente con nuevos compañeros.

PALABRAS CLAVE: Transición de Educación Primaria a Educación Secundaria, educación física, educación deportiva, aprendizaje-servicio. 


\title{
TEACHERS' ENTREPRENEURSHIP IN THE TRANSITION FROM PRIMARY EDUCATION TO SECONDARY EDUCATION: A PROPOSAL FROM SPORT EDUCATION AND SERVICE-LEARNING
}

\begin{abstract}
The transition from Primary Education to Secondary Education is a disturbing and influential period in the school trajectory of more than $50 \%$ of students, with a substantial decrease in self-esteem, academic motivation and academic achievement, as well as the risk of suffering bullying and school dropout. The systems developed to facilitate this process have focused on administrative and organizational procedures, when research shows that the process should focus on social and personal features. In this paper, we expose how the integrated use of Sport Education and Service-Learning can be configured as a powerful tool to combat the problems arising from this process of educational transition. Furthermore, we analyze how the integrated implementation of these two approaches allows to have facilitators of great potential in this process: to have acquaintances who pass with them from primary education to secondary education, to develop the feeling of belonging, to meet older students and to verify that they are friendly, host activities that help them adapt and perceive that the school helps them, and activities in small groups where they can relate closely with new colleagues.
\end{abstract}

KEYWORDS: Transition from primary Education to secondary Education, physical education, sport education, service-learning.

Recibido: 08/12/2018

Aceptado: 28/02/2019

Correspondencia: Luis Miguel García López, Universidad de Castilla - La Mancha, Plaza de la Universidad 3, 02071 Albacete. Email: LuisMiguel.Garcia@uclm.es.

\section{INTRODUCCIÓN}

La transición de Educación Primaria (EP) a Educación Secundaria (ES) es un periodo complejo y difícil en la trayectoria escolar. Los estudiantes, los cuales se hallan en plena adolescencia, tienen que acometer un cambio que viven de manera problemática. En España, hasta un 50\% de los estudiantes sufren problemas en ese proceso de transición (Monarca, Rappoport, y Fernández González, 2012). En este sentido, muchos de estos estudiantes acusan una relevante disminución de su autoestima y motivación académica, lo cual se suele traducir en importantes descensos del rendimiento académico (West, Sweeting y Young, 2010). Esto, aun siendo muy importante, puede verse relegado a un segundo lugar. En muchos casos esta transición de la EP a la ES es el momento en el que se observa cómo se producen conductas de acoso y abandono escolar (Evangelou et al., 2008).

Con el fin de evitar estos problemas generados por la transición de la EP a la ES, las instituciones educativas tienden a desarrollar sistemas para facilitar este proceso, cuyo fundamento suele radicar en los procedimientos administrativos y de organización. Sin 
embargo, las investigaciones realizadas a tal efecto muestran como la base de estas intervenciones deberían ser aspectos sociales y personales (Jindal-Snape y Miller, 2008). En este sentido, se han descrito una serie de facilitadores en este proceso, de los cuales pasamos a describir aquellos que son más destacados. En primer lugar, el hecho de tener conocidos que pasan con ellos de EP a ES suele ser un apoyo muy importante (Weller, 2007). De esta manera, un contexto que puede ser novedoso e intimidador se afronta contando con el apoyo de algunos iguales que son conocidos y sufren las mismas inquietudes y dificultades. En segundo lugar, otro facilitador es desarrollar el sentimiento de pertenencia, de tal manera que el alumno nuevo en un centro pronto se vea reconocido como integrante de este (Cueto, Guerrero, Sugimaru y Zevallos, 2010). Aquí actividades como el deporte, teatro $u$ otras que se realicen en equipo pueden jugar un papel fundamental, pues pertenecer a ese tipo de colectivos puede favorecer esa generación de sentimiento de pertenencia al nuevo centro educativo. En tercer lugar, conocer a alumnos mayores y comprobar que son amistosos, actividades de acogida que les ayuden a adaptarse y percibir que el centro le ayuda, y actividades en grupos reducidos donde puedan relacionarse estrechamente con nuevos compañeros, son sin duda posibilidades de integración que se han mostrado como exitosas (Evangelou et al., 2008).

Una vez expuesto el problema que supone la transición de la EP a la ES, nuestro planteamiento es que existen dos metodologías que, combinadas, tienen un gran potencial para favorecer esta transición. Esto es así debido a que su uso conjunto ofrece como resultado la aplicación de los facilitadores que se han descrito anteriormente. Estas metodologías de enseñanza son el Modelo pedagógico Educación Deportiva (MED) y el Aprendizaje-Servicio (ApS). El objetivo de este trabajo es el análisis de estas dos metodologías de enseñanza de cara a un planteamiento conjunto que tenga un impacto positivo sobre la transición de los alumnos entre EP y ES.

\section{Método}

\section{Procedimiento}

Para el desarrollo de este trabajo nos planteamos analizar los componentes principales de cada una de estas metodologías de enseñanza, describiendo por una parte cuales podrían ser sus contribuciones a la transición entre EP y ES, y por otra parte de qué manera debería hacerse un uso combinado de ambas metodologías. Este análisis se realizará basándonos en los diferentes resultados de las investigaciones realizadas hasta la fecha en relación a ambas metodologías de enseñanza.

\section{Resultados}

\section{El modelo educación deportiva}

A pesar de la creencia popular de que el deporte es educativo per se, la investigación ha demostrado que sólo cuando se utilizan determinadas estrategias y hay un adecuado trabajo del docente de manera sistemática, es cuando se obtienen efectos reales en el desarrollo moral de la persona (Sandford, Armour y Warmington, 2006). 
Dentro de la Educación Física (EF) existen diversos planteamientos de enseñanza que han mostrado ser eficaces a la hora de mejorar diversos componentes de las competencias social y ciudadana, tales como el Aprendizaje Cooperativo (Casey, y Goodyear, 2015) y la Enseñanza de la Responsabilidad Individual y Social (Pozo, Grao-Cruces, y Pérez-Ordás, 2016). Uno de los planteamientos que está obteniendo mejores resultados en la investigación, no sólo por la mejora competencial de los alumnos (García López y González Víllora, 2011), sino por las mejoras motivacionales y de la percepción de eficacia del profesorado, es el MED.

Los modelos de instrucción o modelos pedagógicos en EF (Metzler, 2011) son planteamientos flexibles que mantienen una estructura que el profesor adapta a su contexto de clase. Esta estructura incluye aspectos tales como los contextos de aprendizaje, los aprendices, los contenidos, o las estrategias de enseñanza y evaluación. El MED (Siedentop, 1994) tiene como principal propósito que todos los alumnos, y no solo los mejores, vivan una experiencia deportiva auténtica a través de unidades didácticas de larga duración, donde trabajan en grupos de trabajo o equipos permanentes y asumen roles de responsabilidad dentro de su equipo (entrenador, preparador físico, etc.) y de organización (árbitro, mánager general, etc.). Este trabajo en equipo es uno de los aspectos que antes citábamos y que puede mejorar el sentimiento de pertenencia de los alumnos que pasan a ES (Cueto, Guerrero, Sugimaru y Zevallos, 2010). El MED presta especial atención al desarrollo de valores y la socialización derivados de una práctica deportiva positiva y educativa. Las características esenciales del deporte, junto con estrategias pedagógicas que promueven el desarrollo ético (contratos éticos, juegos modificados, comités éticos), la promoción de la autonomía y responsabilidad personal a través de los roles de equipo y organización, otorgan a este modelo un gran potencial en el desarrollo de valores positivos, tal y como lo ha demostrado la investigación. Así, el MED se ha mostrado eficaz en la mejora de aspectos comportamentales relacionados con los facilitadores necesarios en la transición entre EP y ES. En relación a los aspectos comportamentales, se ha observado que el MED favorece comportamientos más asertivos mediante la reducción de comportamientos pasivos y agresivos (García López, y Gutiérrez, 2015). Esto es importante de cara a que los alumnos presenten menos conductas agresivas, lo cual se podría traducir en un menor número de conductas de acoso hacia los nuevos alumnos. Más aun, en el caso de que se dieran las conductas de acoso y los alumnos las observasen, estas mejoras de la asertividad podrían favorecer que no fueran pasivos, sino que intervinieran a favor de la posible víctima. En segundo lugar, el MED ha tenido resultados positivos en el fomento de comportamientos pro-sociales (García López, Gutiérrez Díaz del Campo, González Víllora, y Valero Valenzuela, 2012; Méndez Giménez, Fernández Río, y Méndez Alonso, 2015). En estos trabajos se ha observado como la participación en temporadas organizadas con el MED ha favorecido la mejora de la relación entre compañeros de la misma clase, favoreciendo la integración de aquellos que sufrían una situación de exclusión dentro del aula. Por tanto, el MED también se muestra como una potente herramienta de cara a que los alumnos de ES perciban a los nuevos alumnos provenientes de EP de manera más positiva y se sientan más proclives a ayudarlos. Por último, el MED se ha mostrado eficaz en el fomento de la resolución de conflictos y la mejora de las habilidades 
comunicativas (Wahl-Alexander, Curtner-Smith, y Sinelnikov, 2016), elementos que son, evidentemente, un medio fundamental a la hora de resolver cualquier problema de convivencia que pueda surgir.

\section{El Aprendizaje-Servicio}

El ApS (del inglés Service-Learning) es una metodología de aprendizaje basada en la puesta en práctica de conocimientos y materias del currículum formal a través de la participación activa de los estudiantes en contextos reales, desarrollando trabajos útiles para la comunidad. Implica un proceso de aprendizaje, reflexión y acción, en el que la propia práctica conduce al aprendizaje (Puig Rovira y Palos Rodríguez, 2006). Esta corriente pedagógica tiene su base en el aprendizaje a través de la experiencia (Dewey, 1985) y la acción educativa que conduce a aprendizajes significativos (Furco, 2011), con un planteamiento que conecta tanto con el constructivismo como con la Escuela Nueva (Álvarez y Sáiz, 2015).

Los principios del ApS son (Anderson, Swick, y Yff, 2001): (a) una alta calidad del servicio a la comunidad; (b) aprendizaje integrado entre el aula y la actividad de servicio; (c) la reflexión realizada por el estudiante que debe permitir la incorporación de experiencias de servicio a las académicas; (d) la opinión de los estudiantes sobre la planificación y la implementación de actividades de servicio debe ser tenida en cuenta para enfatizar su papel de servicio; (e) la colaboración debe asegurar los beneficios para ambas comunidades; y (f) debe existir una evaluación efectiva del progreso, tanto de los aprendizajes como de las metas del servicio realizado.

Esta metodología goza de una creciente implantación en el ámbito educativo, especialmente en la docencia universitaria, en la que se viene constatando la relevancia de proyectos de ApS en el desarrollo de la dimensión social y cívica de los aprendizajes académicos (Puig Rovira, Gijón Casares, Martín García y Rubio Serrano, 2011), propugnada por la UNESCO (1998). En los otros niveles (EP y ES), son recientes y escasas las experiencias documentadas desde una perspectiva de investigación (Martín y Rubio Serrano, 2010). Cabe entender, sin embargo, que esta metodología tiene un gran potencial en este nivel educativo, ofreciendo una experiencia formativa muy valiosa en unos estudiantes que han de recibir una completa educación en valores cívicos y sociales y que se siente estimulado por el desarrollo de actividades de carácter experiencial y contextualizado. Supone este ámbito, por tanto, un área de conocimiento que requiere ser investigada, con las implicaciones que supone de cara a las aportaciones a la comunidad científico-educativa internacional.

\section{El MED como ApS: una vía para la transición de EP a ES}

Los alumnos de ES pueden favorecer la transición de EP a ES de los antiguos alumnos de EP mediante la utilización del ApS y el MED. Ya hemos explicado cómo estos dos planteamientos por separado pueden favorecer aspectos fundamentales para esta transición (como el desarrollo de la empatía, la asertividad y las relaciones sociales, por parte del MED, o la concienciación a la colaboración para la resolución problemas sociales, por parte del ApS) tanto en los alumnos que reciben a los nuevos 
compañeros como los alumnos recibidos. Lo que planteamos en este trabajo es que se generen programas continuos de dos años de duración en los que se establezcan actividades conjuntas entre un centro de ES y los centros de EP que envían sus alumnos a dicho centro de ES una vez acabada la etapa de EP. En Durante el primer año/curso los alumnos de ambas etapas realizarían de manera simultánea una unidad didáctica (temporada) que incluiría diversas actividades compartidas entre todos ellos. Así, durante la fase de pretemporada, los alumnos de ES visitarían los centros de EP para tener un primer contacto con los alumnos de EP, enseñándoles cuestiones relativas a la unidad didáctica que todos ellos están aprendiendo al mismo tiempo. Más adelante, durante la temporada y las fases finales, serían los alumnos de EP los que visitasen el centro de ES, donde de nuevo los alumnos de ES les organizasen la fase final de la competición. En este planteamiento, la unidad didáctica (temporada) que se realizaría de forma común y paralela en los centros de EP y ES, seguiría el MED. Las actividades comunes de servicio de los alumnos de ES hacia los de EP se realizarían conforme a una metodología de ApS.

No vamos a hacer una descripción detallada del MED por falta de espacio (para ello consultar García López y Gutiérrez, 2016). Sin embargo, sí que vamos a tratar de establecer cuáles son los aspectos básicos que permiten al MED, con pequeñas modificaciones, convertirse en ApS.

En primer lugar, es necesario destacar que uno de los fundamentos del MED es la utilización de roles. Esto supone la asunción de una serie de responsabilidades de diferente índole por parte los alumnos, lo cual lleva al desarrollo de su autonomía. En el MED estos roles suponen un servicio a los demás en sí mismo: los entrenadores forman, los árbitros regulan el desarrollo de los partidos, los anotadores registran la información de los partidos, etc. Cuando el MED se integra en una metodología ApS, estos roles sirven para dar además un servicio a otros niños pertenecientes a la comunidad receptora del mismo. Así, pueden ir a colaborar en las funciones de árbitro, entrenador, anotador, etc., ayudándoles a aprender y desempeñar esas funciones.

En segundo lugar, por tanto, el MED utiliza la micro-enseñanza de manera extensiva. Es decir, el profesor delega responsabilidades en alumnos mediante el desarrollo de los roles, de tal manera que los alumnos están asumiendo un aprendizaje, no sólo más activo y participativo, sino que además enseñando aprenden lo que enseñan (Metzler, 2011). En este caso, como los alumnos de ES colaboran en la formación de los roles, están ayudando a la consecución de los aprendizajes intrínsecos a cada rol (habilidades de comunicación, resolución de conflictos...), con lo cual a su vez lo aprenden ellos mismos.

En tercer lugar, la unidad didáctica del MED se estructura como una temporada deportiva ordinaria, de tal manera que tiene tres fases: pretemporada, temporada y fase final. En cada una de estas fases, los alumnos de ES pueden visitar o ser visitados por las comunidades receptoras del servicio. En la fase de pretemporada, los alumnos de ES colaborarán en labores de enseñanza de los contenidos de tipo más deportivo, como pueden ser técnicas y tácticas del deporte que se esté impartiendo. En la fase de temporada regular los alumnos colaborarán en el aprendizaje de los roles, en la propia asunción por parte de los alumnos de la comunidad receptora de las responsabilidades y funciones que representan dichos roles. En la fase final se incluye el 
evento culminante, el cual, a modo del aprendizaje por proyectos, sirve tanto para aplicar todas las competencias desarrolladas (tanto deportivas como organizativas), como para compartir los resultados con la comunidad. Este evento puede establecerse como evento de acogida en el centro de ES, donde los alumnos de ES serán los principales actores de esta acogida.

\section{Conclusiones}

Tal y como indicábamos al explicar el MED, la Educación Física y el Deporte no son educativos per se, sino que es necesaria su implementación de manera adecuada mediante herramientas que hayan sido contrastadas científicamente por docentes suficientemente formados en las mismas. En este trabajo hemos mostrado cómo el uso combinado de las metodologías MED y ApS puede ser un buen ejemplo aplicado a una situación que constituye un grave problema en los actuales sistemas educativos. Esto es así porque el MED es un modelo pedagógico que permite, no solo la mejora de diferentes habilidades sociales que favorecen la convivencia en general, sino la creación de estructuras de enseñanza y aprendizaje que conecten los centros educativos de EP y ES durante el último año de la EP y el primero de la ES. Estas actividades organizadas por los alumnos de ES y tuteladas por los docentes, podrían servir para asegurar que se dieran los condicionantes para una adecuada transición entre la EP y la ES.

Este planteamiento se está llevando actualmente a la práctica en las aulas de 16 centros educativos de Castilla-La Mancha, con la participación de alrededor de 1500 estudiantes y 21 docentes. Esperamos que los resultados obtenidos sirvan para mejorar la transición de los adolescentes de EP a ES.

Este proyecto (referencia EDU 2017-R) ha sido financiado por el Ministerio de Ciencia, Innovación y Universidades y la Agencia Estatal de Investigación como entidad financiadora. Esta cofinanciado por fondos FEDER.

\section{Agradecimientos}

Agradecemos a todos los docentes, alumnos y equipos directivos su participación en el estudio.

\section{REFERENCIAS BIBLIOGRÁFICAS}

Álvarez, C. Á. y Sáiz, G. S. (2015). El aprendizaje servicio y las comunidades de aprendizaje: dos proyectos escolares innovadores que se enriquecen mutuamente. Enseñanza \& Teaching, 33(2), 43-58.

Anderson, J. B., Swick, K. J. y Yff, J. (Eds.). (2001). Service learning in teacher education: Enhancing the growth of new teachers, their students, and communities. Washington, DC: American Association of Colleges for Teacher Education.

Casey, A. y Goodyear, V. A. (2015). Can Cooperative Learning Achieve the Four Learning Outcomes of Physical Education? A Review of Literature. Quest (00336297), 67(1), 56-72. 
Cueto, S., Guerrero, G., Sugimaru, C. y Zevallos, A. M. (2010). Sense of belonging and transition to high schools in Peru. International Journal of Educational Development, 30(3), 277-287.

Dewey, J. (1985). Democracia y escuela. Barcelona: Eumo.

Evangelou, M., Taggart, B., Sylva, K., Melhuish, E., Sammons, P. y Siraj-Blatchford, I. (2008). What Makes a Successful Transition from Primary to Secondary School? London: University of London.

Furco, A. (2011). Service-Learning: A Balanced Approach to Experiential Education. En Taylor, B. y Corporation for National Service (Eds.), Expanding Boundaries: Service and Learning (pp. 66-70). Washington DC: Corporation for national Service.

García-López, L. M. y González-Víllora, S. (2011). La enseñanza deportiva escolar orientada al desarrollo de las competencias básicas. En O. R. Conteras y R. Cuevas (Eds.), Las competencias básicas desde la educación física (pp. 145-160). Barcelona, España: Inde.

García López, L. M. y Gutiérrez Díaz del Campo, D. (2016). Aprendiendo a enseñar deporte. Modelos de Enseñanza Comprensiva y Educación Deportiva. Barcelona: INDE.

García-López, L. M. y Gutiérrez, D. (2015). The effects of a sport education season on empathy and assertiveness. Physical Education \& Sport Pedagogy, 20(1), 1-16.

García López, L. M., Gutiérrez Díaz del Campo, D., González Víllora, S. y Valero Valenzuela, A. (2012). Cambios en la Empatía, la Asertividad y las Relaciones Sociales por la Aplicación del Modelo de Instrucción Educación Deportiva. Revista de Psicologia del Deporte, 21(2), 321-330.

Martín, X. y Rubio Serrano, L. (2010). Prácticas de ciudadanía: diez experiencias de aprendizaje servicio. Barcelona: Octaedro.

Méndez Giménez, A., Fernández Río, J. y Méndez Alonso, D. (2015). Modelo de educación deportiva versus modelo tradicional: efectos en la motivación y deportividad. Revista Internacional de Medicina y Ciencias de la Actividad Física y del Deporte, 15(59), 449-466.

Metzler, M. W. (2011). Instructional models for physical education (3 ed.). Scottsdale: Holcomb Hathaway.

Monarca, H., Rappoport, S. y Fernández González, A. (2012). Factores condicionantes de las trayectorias escolares en la transición entre enseñanza primaria y secundaria. Revista española de orientación y psicopedagogía, 23(3), 257-269.

Pozo, P., Grao-Cruces, A. y Pérez-Ordás, R. (2018). Teaching personal and social responsibility model-based programmes in physical education. European Physical Education Review, 24(1), 56-75. https://doi.org/10.1177/1356336X16664749.

Puig Rovira, J. M. y Palos Rodríguez, J. (2006). Rasgos pedagógicos del aprendizajeservicio. Cuadernos de Pedagogía, 357, 60-63. 
Puig Rovira, J. M., Gijón Casares, M., Martín García, X. y Rubio Serrano, L. (2011). Aprendizaje-servicio y Educación para la Ciudadanía. Revista de Educación, número extraordinario 2011, 45-67.

Sandford, R. A., Armour, K. M. y Warmington, P. C. (2006). Re-engaging disaffected youth through physical activity programmes. British Educational Research Journal, 32(2), 251-271. https://doi.org/10.1080/01411920600569164.

Siedentop, D. (1994). Sport education: quality PE through positive sport experiences. Champaign, III.: Human Kinetics Publishers.

UNESCO (1998). Declaración mundial de la Educación Superior. Recuperado de http://www.unesco.org/education/educprog/wche/declaration_spa.htm.

Wahl-Alexander, Z., Curtner-Smith, M. y Sinelnikov, O. (2016). Influence of a purposefully negotiated season of sport education on one teacher and his pupils. European Physical Education Review, 22(4), 450-464.

Weller, S. (2007). 'Sticking with your Mates?'Children's Friendship Trajectories during the Transition from Primary to Secondary School. Children \& society, 21(5), 339351.

West, P., Sweeting, H. y Young, R. (2010). Transition matters: pupils' experiences of the primary-secondary school transition in the West of Scotland and consequences for well-being and attainment. Research papers in education, 25(1), 21-50. 